\title{
DIFFERENTIAL EXPRESSION OF FGFRS SIGNALING PATHWAY COMPONENTS IN BLADDER CANCER: A STEP TOWARD PERSONALIZED MEDICINE
}

\author{
Ousati Ashtiani Z ${ }^{1,2}$, Tavakkoly-Bazzaz J,*, Salami SA³, \\ Pourmand $\mathrm{MR}^{4}$, Mansouri F ${ }^{5,6}$, Mashahdi $\mathrm{R}^{1}$, Pourmand $\mathrm{G}^{1, *}$
}

\begin{abstract}
*Corresponding Author: Professor Gholamreza Pourmand, Urology Research Center, Sina Hospital, Tehran University Medical Sciences, Hasan Abad Square, Tehran, 113746911, Iran. Tel: +98-216-634-8560. Fax: +98-216-634-8561. Email: pourmand@tums.ac.ir and/ or Associate Professor Javad Tavakkoly-Bazzaz, Department of Medical Genetics, School of Medicine, Tehran University of Medical Sciences, Poursina Street, Tehran, 1417613151, Iran. Tel: +98-218-895-3005. Fax: +98-218-895-3005. Email: tavakkolybazzazj@tums.ac.ir
\end{abstract}

\begin{abstract}
Variations Improper activation and inappropriate expression of fibroblast growth factor receptors ( $F G F R s)$ in cancer suggests that they can act as therapeutic targets. Fibroblast growth factor receptor inhibitors are currently employed in clinical trials of different cancers. Regarding the essence and the importance of the personalized medicine, mainly mirrored by remarkable inter-individual variations in different populations, we aimed to perform a pilot study to address FGFR1 and FGFR3 expression levels and their correlation with the clinicopathological features in Iranian patients with bladder cancer (BC). Paired tumor and adjacent non tumor tissue samples along with their clinico-pathological parameters were obtained from 50 cases diagnosed with $\mathrm{BC}$ in different stages and grades. The mRNA expressions of FGFR1 and FGFR3 in tissue samples were determined by real-time polymerase chain reaction (real-time PCR). The expression levels of FGFR3 were significantly higher in tumor tissues when compared to adjacent normal tissues ( $p=0.007)$, regardless of the stages and grades of the tumor. Over expression was associated with cigarette smoking $(p=0.037)$ and family

\footnotetext{
${ }^{1}$ Urology Research Center, Tehran University of Medical Sciences, Tehran, Iran

2 Department of Medical Genetics, School of Medicine, Tehran University of Medical Sciences, Tehran, Iran Public Health, Tehran University of Medical Sciences, Tehran, Iran

${ }^{5}$ Department of Medical Immunology and Genetics, Faculty of Medicine, Urmia University of Medical Sciences, Urmia, Iran

${ }^{6}$ Cellular and Molecular Research Center, Urmia University of Medical Sciences, Urmia, Iran
}

${ }^{3}$ Department of Biotechnology, University of Tehran, Tehran, Iran

${ }^{4}$ Division of Microbiology, Department of Pathobiology, School of
\end{abstract}

history for cancer $(p=0.004)$. Decreased expression of FGFR1 was observed, remarkably evident in high-grade tumors ( $p=0.047$ ), while over expression was detected in low-grade samples. This pilot study clearly suggests that in Iranian BC patients FGFR 1 and FGFR 3 expression patterns are different, and also highly distinctive with regard to the tumor's stage and grade. Such particular expression patterns may indicate their special values to be employed for interventional studies aiming targeted therapy. Further studies with a larger sample size are needed to validate our results.

Keywords: Bladder cancer (BC); FGFR signaling; FGFR1; FGFR3; Targeted therapy.

\section{INTRODUCTION}

Bladder cancer (BC) is one of the most common types of urinary system cancers in men, and with less frequency, in women worldwide [1]. It is a complex disease resulting from both genetic and environmental factors. Aberrations in different genes' structure and function, molecular derangements, and several environmental factors have been found to play a crucial role in the development of BC. Age, increased body mass index (BMI), occupation, unhealthy diet, and some drugs can increase the risk of BC development [2]. The epigenetic mechanism also involves in $\mathrm{BC}$ tumorigenesis [3]. Blood cancers are generally classified as non muscle invasive with low-grade (pTa/T1) and muscle invasive (pT2-4) that are frequently high-grade tumors [4].

Regulation of different processes during cell cycles, including cell growth, differentiation, cell movement, and apoptosis are orchestrated by diverse signaling pathways. When the above mentioned processes are dysregulated secondary to changes in a key genetic element of cellular homeostasis, tumorigenesis can be the outcome. The fi- 
broblast growth factor receptor (FGFR) signaling pathway has been receiving growing attention as one of the major contributors in cell cycle regulation that in turn, introduces this specific molecule as a potential drug target for cancer therapy [5-7].

The mammalian fibroblast growth factors (FGFs) are a family of growth factors, consisting of 18-22 members that play an essential role in multiple physiological events such as angiogenesis, wound healing, embryonic development, and various endocrine signaling pathways both in health and disease. Fibroblast growth factors signaling dysregulation is evidently present in a considerable number of BC cases [8]. A subfamily of receptor tyrosine kinases (RTKs) named fibroblast growth factor receptor family comprises of four members (FGFR1-4). They are activated by binding to their ligand FGFs, which results in kinase activation. Different changes exemplified by FGFRs mutations and translocations, as well as alterations in mRNA splicing and gene amplification of FGF/FGFR pathway and protein expressions levels have been documented in different cancers [9-14]. Aberrations of the FGFR signaling pathway can activate downstream pathways, PI3K/ AKT, MAPK signaling cascade, those which contribute to tumor progression. The FGFR 1 and FGFR3 mutations and over expression have been reported in $\mathrm{BC}$ [15-18], while FGFR3 alterations were significantly involved in the pathogenesis of urothelial carcinoma (UC) as a whole. However, its clinicopathological implications and significance have not so far been well addressed, especially in the case of muscle-invasive BCs [19]. In contrast to the non muscle invasive $\mathrm{UC}$, where the $F G F R 3$ is frequently mutated or overexpressed, in muscle invasive forms the incidence of FGFR 3 mutation and $\mathrm{mRNA}$ /protein expression changes remain unknown [20]. The FGFRl gene expression alteration is also related to certain cancers $[8,9$, 14]. More notably, a recent study using next generation sequencing in advanced $\mathrm{BC}$ has demonstrated a gene fusion of FGFR1 and NTM (FGFR1-NTM) [21].

Molecular genetic studies on FGFR1 and FGFR3 have revealed the role of these gene changes in different cancers and their value in molecule-targeted therapy. The present study was conducted because of a significant heterogeneity in response of the BC cells to FGFR inhibitors that highlights the importance of the personalized medicine, and also with regard to the remarkable inter-individual variations between different populations. For the first time, this study designed to evaluate FGFR1 and FGFR3 expressions at the mRNA level, and their associations with grade, stage and other clinicopathological features in Iranian subjects with BCs.

\section{MATERIALS AND METHODS}

Patients and Tissue Samples. Paired samples, both bladder tumor and adjacent normal tissue were obtained from 50 Iranian individuals who underwent transurethral bladder tumor resection or radical cystectomy at two university teaching hospitals (Sina and Imam Khomeini Hospitals) in Tehran, Iran. Bladder tumor and non tumor samples from a standard distance were rapidly frozen in liquid nitrogen following collection and stored at $-80{ }^{\circ} \mathrm{C}$ until subsequent RNA extraction.

Of the 50 patients, 43 were males and seven were females. The median age was 66 years, ranging from 33 to 84 years. None of the patients received any treatments, such as Bacillus Calmette-Guerin (BCG) therapy, chemotherapy, which might alter the situation of the FGFR signaling pathway in terms of its status and activity. Clinicopathological information including grade, stage, lymph node metastasis, age, gender, smoking, alcohol use, family history of cancer, was provided for all subjects. In this research, written informed consent was signed by all participants, after being informed about the goals of the study. This study was approved by the Research Review Board and also the Ethics Committee of Tehran University of Medical Sciences (TUMS), Tehran, Iran.

Total RNA from both tumor and adjacent non tumor tissues were isolated using TriPure Isolation Reagent (Roche Life Science, Mannheim, Germany) according to the manufacturer's protocol. The quality and quantity of extracted RNAs were measured by the absorbance ratio at 280/260 nm using NanoDrop-2000 spectrophotometer (Thermo Fisher Scientific, Wilmington, DE, USA). In order to remove possible DNA contamination from RNA, DNase I treatment was performed. The cDNA was synthesized from $1 \mu \mathrm{g}$ RNA by oligo dT, Random 6-mer and reverse transcription Enzyme using PrimeScript ${ }^{\mathrm{TM}}$ RT reagent kit (Takara, Kusatsu, Shiga, Japan) according to the manufacturer's instructions. It was designed to perform optimized reverse transcription-polymerase chain reaction (RT-PCR). Thermal Cycler (Senso Quest GmbH, Göttingen, Germany) was used for the incubation reaction mixture at $37^{\circ} \mathrm{C}$ for $15 \mathrm{~min}$. and $85^{\circ} \mathrm{C}$ for 5 seconds. The cDNAs were stored at $-20{ }^{\circ} \mathrm{C}$ until further use.

For real-time PCR, specific sets of primers were designed for FGFR1, FGFR3 and GAPDH as housekeeping genes. All amplicon lengths for real-time PCR were less than 200 bp long. Primer sets were checked by primerBLAST and Oligoanalyzer software (https://eu.idtdna. com/ calc/analyzer). Table 1 shows the 5 ' $>3$ ' sequence of the primers and amplicon lengths. 
Table 1. List of primer sets for real-time polymerase chain reaction.

\begin{tabular}{|l|l|c|}
\hline Primers & Sequences $(\mathbf{5}>\mathbf{3}$ ') & Amplicon Size \\
\hline FGFR1-F & GAA GAC TGC TGG AGT TAA TAC C & 159 \\
FGFR1-R & TCT TCC AGG GCT TCC AGA AC & 180 \\
\hline FGFR3-F & CCA CTG TCT GGG TCA AGG AT & 159 \\
FGFR3-R & AGG ATG GAG CGT CTG TCA C & 159 \\
\hline GAPDH-F & ATC CTG GGC TAC ACT GAG C & \\
GAPDH-R & CAC CAC CCT GTT GCT GTA G & \\
\hline
\end{tabular}

F: forward; R: reverse.

Table 2. Clinicopathological characteristics of the study subjects.

\begin{tabular}{|c|c|c|}
\hline $\begin{array}{l}\text { Clinicopathological } \\
\text { Parameters }\end{array}$ & $n$ & $\%$ \\
\hline $\begin{array}{l}\text { Age (years): } \\
\leq 66 \\
\quad>66\end{array}$ & $\begin{array}{l}26 \\
24\end{array}$ & $\begin{array}{l}52.0 \\
48.0\end{array}$ \\
\hline $\begin{array}{l}\text { Sex: } \\
\quad \text { males } \\
\text { females }\end{array}$ & $\begin{array}{r}43 \\
7\end{array}$ & $\begin{array}{l}86.0 \\
14.0\end{array}$ \\
\hline $\begin{array}{c}\text { Stage: } \\
\text { I } \\
\text { II } \\
\text { III } \\
\text { IV }\end{array}$ & $\begin{array}{r}16 \\
20 \\
10 \\
4\end{array}$ & $\begin{array}{r}32.0 \\
40.0 \\
20.0 \\
8.0\end{array}$ \\
\hline $\begin{array}{l}\text { Grade: } \\
\text { low } \\
\text { high }\end{array}$ & $\begin{array}{l}14 \\
36\end{array}$ & $\begin{array}{l}28.0 \\
72.0\end{array}$ \\
\hline $\begin{array}{l}\text { Tumor type: } \\
\text { non invasive muscle } \\
\text { muscle invasive }\end{array}$ & $\begin{array}{l}15 \\
35\end{array}$ & $\begin{array}{l}30.0 \\
70.0\end{array}$ \\
\hline $\begin{array}{l}\text { Family history: } \\
\text { bladder cancer } \\
\text { other cancer } \\
\text { no cancer }\end{array}$ & $\begin{array}{r}8 \\
6 \\
36\end{array}$ & $\begin{array}{l}16.0 \\
12.0 \\
72.0\end{array}$ \\
\hline $\begin{array}{l}\text { Smoker: } \\
\quad \leq 10 \\
\quad>10 \\
\text { non smoker }\end{array}$ & $\begin{array}{r}2 \\
32 \\
16\end{array}$ & $\begin{array}{r}4.0 \\
64.0 \\
32.0\end{array}$ \\
\hline Occupational exposure & 27 & 54.0 \\
\hline
\end{tabular}

Real-time PCR was performed using SYBR Premix EX Taq ${ }^{\mathrm{TM}} \mathrm{II}$ (Takara). The reaction mixture was prepared according to the manufacturer's protocol. The cycling conditions were: 10 seconds at $95^{\circ} \mathrm{C}$ (Takara Master does not need longer hold), followed by 40 cycles at $95^{\circ} \mathrm{C}$ for 10 seconds and $60^{\circ} \mathrm{C}$ for the 20 seconds. Amplification reactions were performed in triplicate for each sample. A melting curve was obtained following amplification. No template control (NTC; nuclease-free water) was included in each run. The quantitative PCR (qPCR) analysis was completed using Rotor-Gene Q (Brisbane, Queensland, Australia). Cycle threshold (ct) values were collected for the genes of interest and glyceraldehyde 3-phosphate dehy- drogenase as housekeeping gene during the log phase of the cycle. Results were normalized to the GAPDH as a reference gene. Agarose gel electrophoresis was used to determine the specificity of the RT-PCR reaction products. Gene expression data analysis was carried out using the $2^{-\Delta \Delta C \mathrm{CT}}$ method according to the following formula: $\Delta \mathrm{ct}_{1}=\mathrm{ct}_{\text {target }}-\mathrm{ct}_{\text {houseckeping }}, \Delta \mathrm{ct}_{2}=\mathrm{ct}_{\text {normal }}-\mathrm{ct}_{\text {houseckeping }}, \Delta \Delta \mathrm{ct}=\Delta \mathrm{ct}_{1}-\Delta \mathrm{ct}_{2}$.

Statistical Analysis. Statistical analysis was performed by the the Statistical Package for Social Sciences (SPSS) version 21 (IBM SPSS Inc., Chicago, IL, USA). The Kolmogorov-Smirnov test was used to assess the normality of quantitative data. Comparison of normalized expression between tumor and non tumor tissues was done using the parametric $t$-test for FGFR3 and nonparametric Mann-Whitney test for FGFR1. A multivariate linear regression analysis was performed to find the relationship between expression and clinicopathological parameters as independent variables by a stepwise method in the model. In these tests, a $p$ value of $\leq 0.05$ was considered to indicate a significant difference.

\section{RESULTS}

Patient Characteristics. This study comprised 50 BC patients including $43(86.0 \%)$ men and seven $(14.0 \%)$ women. Analyses of the subjects showed that $36(72.0 \%)$ cases had high-grade and 14 (28.0\%) had low-grade tumors. Their median age was 66 years (range 33-84 years). There were 34 patients $(68.0 \%)$ with a smoking habit, of which $94.0 \%$ (32/34) had been cigarette smokers for $\geq 10$ years and $20.0 \%(10 / 50)$ were opium addicts. Of these subjects, $16.0 \%(8 / 50)$ had diabetes and $46.0 \%(23 / 50)$ showed cardiovascular and/or respiratory diseases. The rate of occupational exposure was about 54.0\% (27/50). Table 2 shows the demographic and clinicopathological characteristics of all subjects.

Increased mRNA expression of FGFR 3 was observed in $92.0 \%(46 / 50)$ of tumors. Analysis by a multi variable regression method with a stepwise selection variable demonstrated that there was significant association between 
increased expression of FGFR 3 and cigarette smoking ( $p$ $=0.037)$ and family history of cancer $(p=0.004)$ (Table 3 ). We could not find a significant relationship between FGFR3 mRNA over expression and age $(p=0.094)$ and other relevant clinicopathological parameters including stage, grade and types of the tumor. The comparison of normalized expression in tumor and non tumor tissues showed significant difference (fold change 5.7; $p=0.01$ ) (Figure 1).

Unexpectedly, FGFR1 mRNA expression in our BC cases was decreased in $60.0 \%(30 / 50)$ of samples. The majority of high grade tumor (75.0\%) (27/36) reflected a significant association with decreased level of FGFR1 mRNA expression $(p=0.047)$. About 64.2\% (9/14) of low-grade tumors showed increased expression of FGFR1. There was also a significant association between normalized expression in tumor $v s$. non tumor tissue (fold change $-4.5 ; p=0.01$ ) (Figure 2). Other clinicopathological parameters did not represent any significant difference with FGFR1 expression.

\section{DISCUSSION}

This is the first study that demonstrates FGFR1 and FGFR3 mRNA expression levels in Iranian patients with BCs. The FGF/FGFR pathway is one of the central mecha- nisms that govern differentiation, proliferation, survival and many other issues of cellular characteristics. Large number of evidence supports the appropriate functionality of FGFs-FGFRs systems is dysregulated at any point within their signaling cascade that could frequently lead to cancer development via activation of the downstream pathway [22].

The FGFs and FGFRs changes at different levels featured by gene mutations, gene fusions and aberrant expressions or amplification have been identified in the progression of UCs [8,21]. Such aberrations in FGFR3 are one of the most common molecular events in UCs, but some of them such as gene amplification are relatively rare $[23,24]$. Preclinical studies weighed the applicability of FGFR3 for targeted therapy [25]. In this pilot study, FGFR3 mRNA over expression was observed in the majority of all subjects (shaped mainly by high grade or muscle invasive tumors) $(92.0 \%$ ), while most of the previous studies were conducted in cases with non muscle invasive tumors $[20,26]$. The frequency of FGFR3 mRNA over expression between the subjects of the present study was clearly higher than that of previous reports in BC [20]. Analyses of our data showed that FGFR3 mRNA over expression was associated with smoking and family history ( $p=0.037, p=0.004$, respectively). This FGFR3 tumoral over expression was not related to the other clinicopatho-

Table 3. Multivariate linear regression analysis of FGFR1 and FGFR3 expressions with clinicopathological variables.

\begin{tabular}{|l|l|c|c|c|c|}
\hline $\begin{array}{l}\text { Relative } \\
\text { Expression }\end{array}$ & Variable(s) & $\begin{array}{c}\text { Undersized } \\
\text { Coefficients B }\end{array}$ & Standard Error B & $\boldsymbol{t}$-Test & $\boldsymbol{p}$ Value \\
\hline FGFR1 & no significant variable & & & & \\
\hline FGFR3 & (constant) & -28.908 & 11.031 & -2.621 & 0.021 \\
& family history of BC & 14.266 & 4.125 & 3.458 & 0.004 \\
& cigarette smoking & 11.568 & 4.968 & 2.329 & 0.037 \\
\hline
\end{tabular}

BC: bladder cancer.

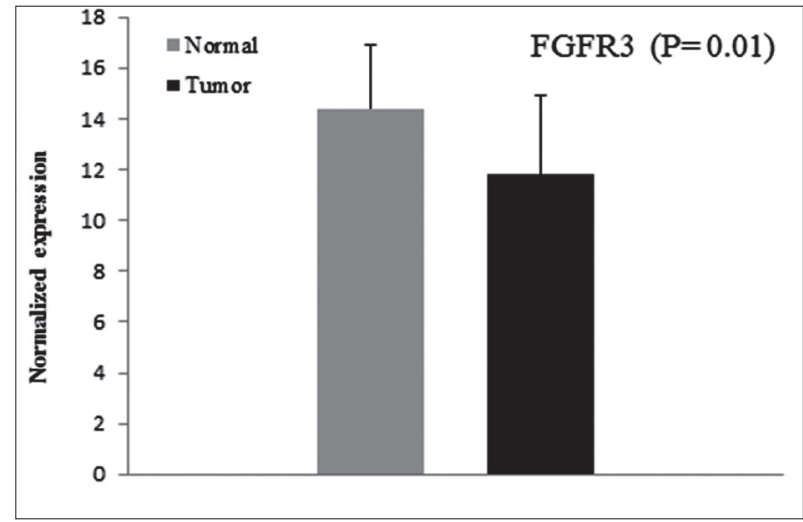

Figure 1. Normalized expression of FGFR3 in cancerous and normal tissues.

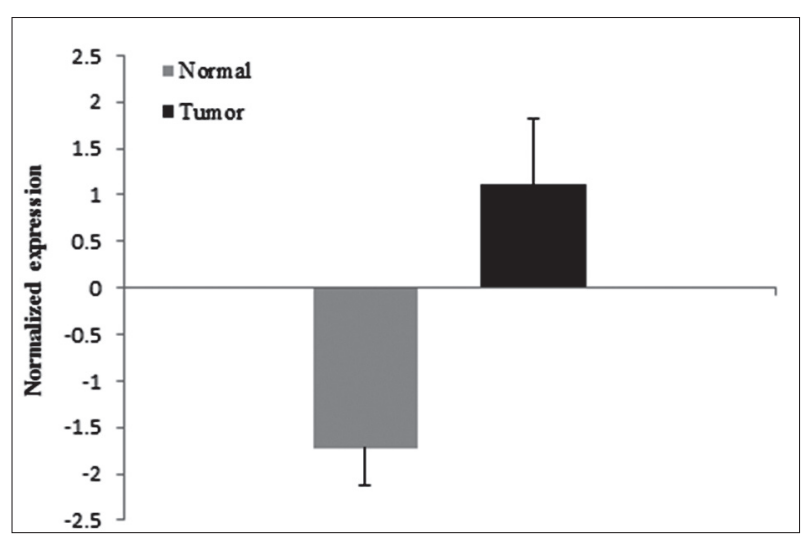

Figure 2. Normalized expression of FGFR1 in cancerous and normal tissues. 
logical parameters including tumor grade or stage. Since up- and down-regulation of FGFRs have been found in the context of different cancers $[10,19,28,29]$, it is suggested that the potential oncogenic and tumor suppressive influencing FGFRs can be implemented by either its up- or down-regulation according to the nature and stages/grades of the tumors. Mainly mediated by FGFR3 gene mutations, over expression of FGFR 3 mRNA and protein levels have been evident frequently in different cancers, which may suggest more clearly its oncogenic properties [10,22,27]. In the current study, increased expression of FGFR3 in most of the tumor tissues, regardless of their grade, stage and type, suggest the importance of the FGFR3 role as a driving member for tumor growth and progression in Iranian BC subjects.

In terms of FGFR 1 expression patterns, to some extent, our results are not consistent with previous reports. We observed decreased mRNA expression of FGFR1 in $60.0 \%$ of cases, of which the majority of them had highgrade $\mathrm{BC}(p=0.01)$, while previous studies found an increased expression of both mRNA and protein in mostly non invasive BC $[9,20,24,29]$. However, in another study that was conducted on an invasive form of $\mathrm{BC}$, the level of FGFR1 was significantly up-regulated [18]. This discrepancy may be partly attributed to geographical and methodological parameters including technical issues as well as histopathological heterogeneity within individual tumor type and also proportion of case within various grade and stage classes.

While the pivotal role of the FGFR1 gene in the development of different types of cancer is suggested by several studies, it seems the underlying mechanism for FGFR1 gene involvement or the pattern of its dysregulated expression is not fixed. For example, it is reported that the main reason for FGFR 1 up-regulation in breast cancer is the amplification of the FGFR1 gene at the genome level that could be evidenced by high FGFRl expression level in breast tumors [30], whereas we mentioned before, it seems that the amplification of FGFRs genes is not the case in $\mathrm{BC}$. In addition to $\mathrm{BC}$, the reduced expression of FGFR1 is also evident in some other types of cancers, such as parathyroid cancer [14]. Such decreased expression patterns, which may suggest a potential tumor suppressive role for FGFRs, was also reported for FGFR2 in several cancers including bladder, liver, salivary gland and prostate; but the exact mechanism remains unknown [22].

However, it is so complicated to find out which member of the FGFRs system is playing the central role in cancer. Cheng et al. [29] stated that FGFR3 plays a more important role than FGFRlin stimulating BC tumor cell proliferation. Our data indicates that reduced mRNA expression of FGFR1 is associated with high-grade tumors, contrasted by its increased expression in low-grade tumors. This would suggest that although FGFRl is less important than FGFR3 in BC cell proliferation, up-regulation of FGFR1 expression can be considered as an early event, the same as FGFR3, in the initiation and development of $\mathrm{BC}$ that was also raised by some previous reports [18,31]. In this regard, increased expression of FGFR 1 and FGFR3 proteins are reported in the early stages of non small cell lung cancer [13].

In addition, it seems that FGFRs act differentially depending on the tumor's type that could explain that contradictory results may be partly due to the cell type specificity. For instance, in cervical cancer, expression of FGFR1, FGFR2 and FGFR4 were higher in cancerous tissues, whereas FGFR3 was higher in non cancerous tissues [27]. In addition to cell specificity, alternative splicing of the extracellular fragment of FGFR1-3 may be partly accountable for this discrepancy. Alternative splicing can result in the formation of isoforms, which in turn, change ligand-binding specificity and the switching of the cross-talk between isoforms. This, in turn, causes alterations in FGFR signaling leading to influences on downstream signaling cascades. It has been reported that two splice variants of FGFR1 gene called FGFR $1 \alpha$ and FGFR1 $\beta$ were expressed at similar levels in normal urothelial cells, but expression level of FGFR1 $\beta$ in tumor cells were higher than in controls. They also presented that FGFR1 $\beta$ : FGFR $1 \alpha$ ratio was significantly increased in relation to tumor stage and grade (31). On the other hand, the epigenetic process alterations are thought to influence gene expression largely at the transcription level [32], it may be considered as a reason for this inconsistency in different studies.

To the best of our knowledge, this pilot study is the first report examining the expression of two components of FGFR signaling pathway (FGFR1, FGFR3) in Iranian $\mathrm{BC}$ subjects in order to find out whether they can be used as a molecule for targeted interventions. The present study showed that lower mRNA expression of FGFR1 was significantly associated with high-grade tumors and in low-grade tumors increased expression was observed. Our results suggest that the expression mode of FGFR1 is somehow related to the cancer grade. With regard to FGFR1 over expression in low-grade tumors and FGFR3 over expression in both high- and low-grade tumors, it is probable that these components of FGFR signaling cascade may be considered distinctly as potential candidates for targeted therapy. 
However, under individualized medicine strategy for $\mathrm{BC}$ in the future, it seems that FGFRs could provide attractive targets for therapeutic interventions. Because of the heterogeneity between different populations, such objective requires a more comprehensive understanding on the role of FGFRs in $\mathrm{BC}$, coupling with the data on larger sample sizes of the cases and controls. Finally, it is very crucial to remember that the inter-individual phenotypic variation at the cellular and molecular level is not solely dictated by genetic impressions. Accordingly, epigenetic issues, which themselves are influenced by environmental factors, should be taken into account to unravel the reality behind these observations.

\section{ACKNOWLEDGMENTS}

We would like to thank Mrs. Fariba Heidari and Mrs. Goli Abdi [Urology Research Center, Tehran University of Medical Sciences, Tehran, Iran], for their contribution in administrative issues.

Declaration of Interest. This research was supported by a grant from Tehran University of Medical Sciences (TUMS) [Grant No. 26562].The authors report no conflicts of interest. The authors alone are responsible for the content and writing of this article.

\section{REFERENCES}

1. Rodriguez-Vida A, Saggese M, Hughes S, Rudman S, Chowdhury S, Smith NR, et al. Complexity of FGFR signalling in metastatic urothelial cancer. J Hematol Oncol. 2015; 8: 119. doi: 10.1186/s13045-015-0221-6.

2. Knowles MA, Hurst CD. Molecular biology of bladder cancer: New insights into pathogenesis and clinical diversity. Nat Rev Cancer. 2015; 15(1): 25-41.

3. Li H-T, Duymich CE, Weisenberger DJ, Liang G. Genetic and epigenetic alterations in bladder cancer. Inter Neurourol J. 2016; 20(Suppl 2): S84-S94.

4. Nagata M, Muto S, Horie S. Molecular biomarkers in bladder cancer: Novel potential indicators of prognosis and treatment outcomes. Dis Markers. 2016; 2016: 8205836. doi: 10.1155/2016/8205836.

5. Martin GS. Cell signaling and cancer. Cancer Cell. 2003; 4(3): 167-174.

6. Ornitz DM, Itoh N. The fibroblast growth factor signaling pathway. WIREs Dev Biol. 2015; 4(3): 215-266.

7. Wesche J, Haglund K, Haugsten EM. Fibroblast growth factors and their receptors in cancer. Biochem J. 2011; 437(2): 199-213.
8. di Martino E, Tomlinson DC, Knowles MA. Adecade of FGF receptor research in bladder cancer: Past, present, and future challenges. Adv Urol. 2012; 2012 : 429213. doi: 10.1155/2012/429213.

9. Lim S, Koh MJ, Jeong HJ, Cho NH, Choi YD, Cho do Y, et al. Fibroblast growth factor receptor 1 overexpression is associated with poor survival in patients with resected muscle invasive urothelial carcinoma. Yonsei Med J. 2016; 57(4): 831-839.

10. Dienstmann R, Rodon J, Prat A, Perez-Garcia J, Adamo B, Felip E, et al. Genomic aberrations in the FGFR pathway: Opportunities for targeted therapies in solid tumors. Ann Oncol. 2014; 25(3): 552-563.

11. Wang Z, Zhang C, Sun L, Liang J, Liu X, Li G, et al. FGFR3, as a receptor tyrosine kinase, is associated with differentiated biological functions and improved survival of glioma patients. Oncotarget. 2016; 7(51):98587-84593.

12. Yashiro M, Matsuoka T. Fibroblast growth factor receptor signaling as therapeutic targets in gastric cancer. World J Gastroenterol. 2016; 22(8): 2415-2423.

13. Theelen WS, Mittempergher L, Willems SM, Bosma AJ, Peters DD, van der Noort V, et al. FGFR1, 2 and 3 protein overexpression and molecular aberrations of FGFR3 in early stage non-small cell lung cancer. J Pathol Clin Res. 2016; 2(4): 223-233.

14. Yan J, Jingbo C, Wang D, Xie S, Yuan L, Zhong X, et al. A correlation between decreased parathyroid alpha-Klotho and fibroblast growth factor receptor 1 expression with pathological category and parathyroid gland volume in dialysis patients. Inter Urol Nephrol. 2015; 47(4): 701-706.

15. Serizawa RR, Ralfkiær U, Steven K, Lam GW, Schmiedel S, Schüz J, et al. Integrated genetic and epigenetic analysis of bladder cancer reveals an additive diagnostic value of FGFR3 mutations and hypermethylation events. Inter J Cancer. 2011; 129(1): 78-87.

16. Kompier LC, Lurkin I, van der Aa MN, van Rhijn BW, van der Kwast TH, Zwarthoff EC. FGFR3, HRAS, KRAS, NRAS and PIK3CA mutations in bladder cancer and their potential as biomarkers for surveillance and therapy. PLoS One. 2010; 5(11): e13821.

17. Juanpere N, Agell L, Lorenzo M, De Muga S, LópezVilaró L, Murillo R, et al. Mutations in FGFR3 and PIK3CA, singly or combined with RAS and AKT1, are associated with AKT but not with MAPK pathway activation in urothelial bladder cancer. Hum Pathol. 2012; 43(10): 1573-1582. 
18. Abdul-Maksoud RS, Shalaby SM, Elsayed WS, Elkady S. Fibroblast growth factor receptor 1 and cytokeratin 20 expressions and their relation to prognostic variables in bladder cancer. Gene. 2016; 591(2): 320-326.

19. Sung JY, Sun JM, Chang Jeong B, Il Seo S, Soo Jeon $\mathrm{S}$, Moo Lee $\mathrm{H}$, et al. FGFR3 overexpression is prognostic of adverse outcome for muscle-invasive bladder carcinoma treated with adjuvant chemotherapy. Urol Oncol. 2014; 32(1): 49.e23-e31.

20. Guancial EA, Werner L, Bellmunt J, Bamias A, Choueiri TK, Ross R, et al. FGFR3 expression in primary and metastatic urothelial carcinoma of the bladder. Cancer Med. 2014; 3(4): 835-844.

21. Bertz S, Abee C, Schwarz-Furlan S, Alfer J, Hofstadter F, Stoehr R, et al. Increased angiogenesis and FGFR protein expression indicate a favourable prognosis in bladder cancer. Virchows Archiv. 2014; 465(6): 687-695.

22. Ahmad I, Iwata T, Leung HY. Mechanisms of FGFR-mediated carcinogenesis. Biochim Biophys Acta. 2012; 1823(4): 850-860.

23. Netto GJ. Molecular biomarkers in urothelial carcinoma of the bladder: are we there yet? Nat Rev Urol. 2011; 9(1): 41-51.

24. Fischbach A, Rogler A, Erber R, Stoehr R, Poulsom $\mathrm{R}$, Heidenreich A, et al. Fibroblast growth factor receptor (FGFR) gene amplifications are rare events in bladder cancer. Histopathol. 2015; 66(5): 639-649.

25. Lamont FR, Tomlinson DC, Cooper PA, Shnyder SD, Chester JD, Knowles MA. Small molecule FGF receptor inhibitors block FGFR-dependent urothelial carcinoma growth in vitro and in vivo. Brit J Cancer. 2011; 104(1): 75-82.

26. Maeng YH, Eun SY, Huh JS. Expression of fibroblast growth factor receptor 3 in the recurrence of nonmuscle-invasive urothelial carcinoma of the bladder. Korean J Urol. 2010; 51(2): 94-100.

27. Choi CH, Chung JY, Kim JH, Kim BG, Hewitt SM. Expression of fibroblast growth factor receptor family members is associated with prognosis in early stage cervical cancer patients. J Transl Med. 2016; 14(1): 124.

28. Zhou W, Du X, Song F, Zheng H, Chen K, Zhang W, et al. Prognostic roles for fibroblast growth factor receptor family members in malignant peripheral nerve sheath tumor. Oncotarget. 2016; 7(16): 22234-22244.

29. Cheng T, Roth B, Choi W, Black PC, Dinney C, McConkey DJ. Fibroblast growth factor receptors-1 and -3 play distinct roles in the regulation of bladder cancer growth and metastasis: Implications for therapeutic targeting. PLoS One. 2013; 8(2): e57284.

30. Chen Y, Xie X, Li X, Wang P, Jing Q, Yue J, et al. FGFR antagonist induces protective autophagy in FGFR1-amplified breast cancer cell. Biochem Biophys Res Commun. 2016; 474(1): 1-7.

31. Tomlinson DC, Knowles MA. Altered splicing of FGFR1 is associated with high tumor grade and stage and leads to increased sensitivity to FGF1 in bladder cancer. Am J Pathol. 2010; 177(5): 2379-2386.

32. Gibney ER, Nolan CM. Epigenetics and gene expression. Heredity (Edinb). 2010; 105(1): 4-13. 